\title{
Global and Targeted miRNA Expression Profiling in Clear Cell Renal Cell Carcinoma Tissues Potentially Links miR-155-5p and miR-210-3p to both Tumorigenesis and Recurrence
}

Jinhua Zhang, ${ }^{\dagger \dagger}$ Yuanqing Ye, ${ }^{*}$ David W. Chang, ${ }^{*}$ Shu-Hong Lin, ${ }^{*}$ Maosheng Huang, ${ }^{*}$ Nizar M. Tannir, ${ }^{\ddagger}$ Surena Matin, Jose A. Karam, ${ }^{\S}$ Christopher G. Wood, ${ }^{\S}$ Zhi-Nan Chen, ${ }^{\dagger \uparrow}$ and Xifeng Wu*

From the Departments of Epidemiology, * Genitourinary Medical Oncology, ${ }^{\ddagger}$ and Urology, ${ }^{\S}$ The University of Texas MD Anderson Cancer Center, Houston, Texas; the College of Life Sciences and Bioengineering, ${ }^{\dagger}$ School of Science, Beijing Jiaotong University, Beijing, China; and the Cell Engineering Research Center and Department of Cell Biology," State Key Laboratory of Cancer, Fourth Military Medical University, Xi'an, China

\author{
Accepted for publication \\ July $10,2018$. \\ Address correspondence to \\ Xifeng Wu, M.D., Ph.D., \\ Department of Epidemiology, \\ Unit 1340, The University of \\ Texas MD Anderson Cancer \\ Center, 1515 Holcombe Blvd., \\ Houston, TX 77030. E-mail: \\ xwu@mdanderson.org.
}

\begin{abstract}
About $30 \%$ of patients undergoing nephrectomy for renal cell carcinoma (RCC) experience disease recurrence. We profiled miRNAs dysregulated in clear-cell (cc) RCC tumor tissues and predictive of recurrence. The expression levels of 800 miRNAs were assessed in paired tumor and normal tissues from a discovery cohort of 18 cCRCC patients. miRNAs found to be differentially expressed were examined in a validation set of 205 patients, using real-time quantitative PCR. Tumor-normal data from 64 patients in The Cancer Genome Atlas were used for external validation. Twenty-eight miRNAs were consistently dysregulated in tumor tissues. On dichotomized analysis, patients with high levels of miR-155-5p and miR-210-3p displayed an increased risk for ccRCC recurrence (hazard ratio, 2.64; 95\% CI, 1.49 to 4.70; $P=0.0009 ;$ and hazard ratio, $1.80 ; 95 \% \mathrm{CI}, 1.04$ to $3.12 ; P=0.036$, respectively) and a shorter median recurrence-free survival time than did patients with low levels $[P<0.01$ (log rank test)]. A risk score was generated based on the expression levels of miR-155-5p and miR-210-3p, and the trend test was significant $(P=0.005)$. On pathway analysis, target genes regulated by miR-155-5p and miR-210$3 p$ were mainly enriched in inflammation-related pathways. We identified and validated multiple miRNAs dysregulated in cCRCC tissues; miR-155-5p and miR-210-3p were predictive of ccRCC recurrence, pointing to potential utility as biomarkers and underlying biological mechanisms. (Am J Pathol 2018, 188: 2487-2496; https://doi.org/10.1016/j.ajpath.2018.07.026)
\end{abstract}

Renal cell carcinoma (RCC) accounts for about $3 \%$ of all malignant tumors in adults. ${ }^{1}$ Clear-cell (cc) RCC is the most common histologic subtype of RCC, accounting for about $70 \%$ to $80 \%$ of cases. ${ }^{2}$ Although progress has been made in cancer-diagnostic technology, particularly in abdominal imaging, more than half of RCC cases are still detected incidentally. ${ }^{3}$ As a result, $20 \%$ to $30 \%$ of patients are found to have metastatic disease at the time of diagnosis. ${ }^{4}$ Moreover, the prognosis after nephrectomy remains unsatisfactory. Around $30 \%$ of patients who undergo nephrectomy experience local recurrence or progression to metastasis. ${ }^{5}$ Even in patients whose disease shows similar clinical and pathologic features, outcomes might differ widely. Therefore, reliable predictors of RCC clinical outcomes are urgently needed. ${ }^{6}$

miRNAs are 19- to 25-nucleotide, noncoding RNAs that regulate gene expression by targeting mRNA for the degradation or repression of translation. ${ }^{7}$ A variety of biological functions can be affected by miRNAs, including cell growth, apoptosis, and differentiation. Because these

Supported in part by NIH grant R01 CA170298 (X.W.) and the Center for Translational and Public Health Genomics, Duncan Family Institute for Cancer Prevention, The University of Texas MD Anderson Cancer Center (institutional funding; X.W.).

Disclosures: None declared. 
functions are often dysregulated in cancer, researchers have sought to determine the role of miRNAs in tumor development and progression. ${ }^{8}$ Indeed, aberrant expression of miRNAs has been found in multiple cancers, including RCC. Evidence suggests that some of these miRNAs would be promising novel biomarkers of oncogenesis, progression, and clinical outcomes.

So far, several studies have examined the dysregulation of miRNAs in RCC tumor tissues (Supplemental Table S1) ${ }^{9-13}$ and assessed the association of miRNA expression with RCC clinical outcomes (Supplemental Table S2). ${ }^{9,14-18}$ However, only a few miRNAs have been consistently shown to be altered, including miR-210-3p, miR-215-5p, miR-200c-3p, and miR-532-5p (Supplemental Table S1). These miRNAs might play important roles in the pathogenesis of ccRCC. Additionally, several miRNAs, including miR-106b, miR204-5p, and miR-139-5, have been shown to be associated with RCC recurrence (Supplemental Table S2). Further replication of these results is necessary. The inconsistencies among these published studies may have been attributable to variations in study population, sample size, sample selection, miRNA assay method, and detection sensitivity. Furthermore, our understanding of miRNome is constantly improving, and the list of identified miRNAs is expanding. Currently, 1881 precursor miRNAs and 2588 mature miRNAs are identified in the miRBase database. ${ }^{19}$

In this multiphase study, a global profiling of miRNA expression in paired tumor-normal tissues from ccRCC patients was first performed using the NanoString platform, and the results were then validated by targeted real-time quantitative PCR TaqMan assay in a separate group of ccRCC patients. In addition, publicly available data from The Cancer Genome Atlas (TCGA) were analyzed to identify dysregulated miRNAs that have potential roles in cancer development. Second, the association of dysregulated miRNAs with the risk for recurrence was evaluated, aiming to identify miRNAs that may serve as novel biomarkers to classify patients at high risk for ccRCC recurrence.

\section{Materials and Methods}

\section{Study Population and Sample Collection}

The study population included 223 white patients with newly diagnosed and histologically confirmed ccRCC, recruited from The University of Texas MD Anderson Cancer Center (Houston, TX; MDA) between 2001 and 2014. The patients' tumors were staged according to the criteria in the sixth edition of the American Joint Committee on Cancer's staging manual. A detailed description of the study population was published previously. ${ }^{20}$ Recruitment was not restricted by age, sex, or cancer stage. Patients' lifestyle and demographic information, including tobacco and alcohol use history, weight and height for calculating body mass index, and medical history, were collected from in-person interviews. Clinical data regarding disease stage, histologic grade, tumor size, treatment, and follow-up information were abstracted from medical records.

The study flowchart is shown in Supplemental Figure S1. The discovery set included paired tumor and normal tissues from 18 patients ( 9 of whom experienced tumor recurrence after resection, and 9 of whom did not). Tumor-normal tissue pairs from 205 patients (63 with tumor recurrence and 142 without recurrence) were used in the validation phase. An independent data set consisting of $68 \mathrm{ccRCC}$ tumor and adjacent normal-tissue pairs was downloaded from TCGA and used as an external validation set (The Genomic Data Commons Data Portal, https://portal.gdc. cancer.gov, data retrieved on January 28, 2016). For the validation phase, ccRCC patients with recurrence were frequency-matched, by age, sex, and clinical stage, to patients without recurrence. Due to the small number of minority groups, only non-Hispanic white patients were included in the study. The MDA IRB approved the protocol of this study, and all participating patients provided written informed consent.

Fresh tumor and paired adjacent normal-tissue specimens were resected from the $223 \mathrm{ccRCC}$ patients by experienced surgeons. The specimens were frozen in liquid nitrogen within 10 minutes of resection and were then stored at $-80^{\circ} \mathrm{C}$ until RNA extraction.

\section{RNA Extraction}

Total RNAs were isolated from the 223 pairs of ccRCC tumor and adjacent normal tissues, using the miRNeasy Mini Kit (Qiagen, Hilden, Germany) according to manufacturer's protocol. The concentration and the purity of the RNAs were evaluated using a NanoDrop ND-100 spectrophotometer (Thermo Fisher Scientific, Waltham, MA).

\section{miRNA Profiling}

The expression profiles of 800 human miRNAs were generated using the NanoString nCounter assay (NanoString Technologies, Seattle, WA) in 18 pairs of ccRCC tumor and adjacent normal-tissue samples (9 pairs each from patients with recurrence and nonrecurrence) in the discovery set. In total, 80 to $100 \mathrm{ng}$ of RNA was used for the nCounter miRNA sample-preparation reactions. All samples were prepared according to the manufacturer's instructions. Preparation of small RNA samples involved the ligation of a specific DNA tag onto the $3^{\prime}$ end of each mature miRNA. These tags were designed to normalize the melting temperatures of the miRNAs and to provide a unique identification for each miRNA species in the sample. After hybridization and removal of excess capture and reporter probes, the purified ternary complexes that were bound to the imaging surface were elongated and immobilized. The surface was then imaged using the nCounter digital analyzer. The data were normalized to the mean 
expression level of the 100 most-expressed miRNAs across all samples. Both raw and processed NanoString miRNA array data with accompanied metadata files were uploaded and submitted to the Gene Expression Omnibus repository (https://www.ncbi.nlm.nih.gov/geo; accession number GSE116251).

\section{Validation of miRNA Expression Using Real-Time Quantitative PCR Array}

The Fluidigm 96.96 Dynamic Array (Fluidigm Corp., San Francisco, CA) and Biomark HD detection system (Fluidigm Corp.) were used for miRNA quantification, according to the manufacturer's protocol, in the 205 pairs of ccRCC tumor and adjacent normal tissues (63 pairs with recurrence and 142 pairs with nonrecurrence) in the validation cohort.

Purified RNA samples were reverse-transcribed using the TaqMan MicroRNA Reverse Transcription Kit (Applied Biosystems, Foster City, CA), 150 ng of total RNA, and pools of Megaplex RT Primers (Thermo Fisher), followed by a preamplification step using Megaplex PreAmp Primers (Thermo Fisher). The expression levels of miRNAs were normalized to that of the snRNA U48, and the $2^{-\Delta \Delta \mathrm{Ct}}$ method was used for the analysis. ${ }^{21,22}$ miRNAs detected in at least $80 \%$ of the tissue samples were included for further analyses. Fifty-six miRNAs that were most differentially expressed in tumor and adjacent normal tissues from the recurrence and nonrecurrence groups in the discovery set $(P<0.01$; fold change, $>1.4)$ were evaluated in the validation set. From these miRNAs, eight (miR-155-5p, miR-18a-5p, miR-192-5p, miR-204-5p, miR-210-3p, miR27b-3p, miR-28-5p, and miR-34a-5p) that showed differential expression in tumor and adjacent normal tissues were further selected on the basis of the comparison between the recurrence and nonrecurrence groups.

\section{Validation in the TCGA Data Set}

Thirty-one candidate miRNAs that were differentially expressed with significance $(P<0.05)$ and exhibited the same pattern of up- or down-regulation in the MDA discovery and validation data sets were further validated in the TCGA data set.

\section{Pathway Analysis}

Analysis of potential target genes and enriched pathways was conducted with a web-based analytical tool, miRsystem (http://mirsystem.cgm.ntu.edu.tw, last accessed December 15, 2017), ${ }^{23}$ which offers comprehensive data on predicted and validated miRNA targets from five different data sets. $P$ values of $<0.05$ were considered statistically significant.
Table 1 Host Characteristics of the ccRCC Study Populations at MDA and in the TCGA Database

\begin{tabular}{|c|c|c|c|}
\hline Characteristics & $\begin{array}{l}\text { Discovery } \\
\text { (MDA) } \\
(n=18)\end{array}$ & $\begin{array}{l}\text { Validation } \\
\text { (MDA) } \\
(n=205)\end{array}$ & $\begin{array}{c}\text { Validation } \\
\text { (TCGA) } \\
(n=61)\end{array}$ \\
\hline $\begin{array}{l}\text { Age in years, } \\
\text { median (range) }\end{array}$ & $72(57-78)$ & $58(28-85)$ & $62(38-86)$ \\
\hline \multicolumn{4}{|l|}{ Sex, $n(\%)$} \\
\hline Male & $11(61.1)$ & $124(60.5)$ & $45(73.8)$ \\
\hline Female & $7(38.9)$ & $81(39.5)$ & $16(26.2)$ \\
\hline \multicolumn{4}{|l|}{ Stage, $n(\%)$} \\
\hline $\mathrm{I}$ & $4(22.2)$ & $110(53.7)$ & $20(32.8)$ \\
\hline II & $1(5.6)$ & $15(7.3)$ & $10(16.4)$ \\
\hline III & $13(72.2)$ & $58(28.3)$ & $13(21.3)$ \\
\hline IV & $0(0)$ & $22(10.7)$ & $18(29.5)$ \\
\hline \multicolumn{4}{|l|}{ Grade, $n(\%)$} \\
\hline 1 & $0(0)$ & $12(5.9)$ & $23(37.7)$ \\
\hline 2 & $5(27.8)$ & $69(33.7)$ & $25(41.0)$ \\
\hline 3 & $8(44.4)$ & $90(43.9)$ & $13(21.3)$ \\
\hline 4 & $4(22.2)$ & $32(15.6)$ & $0(0)$ \\
\hline Unknown & $1(5.6)$ & $2(1.0)$ & $0(0)$ \\
\hline \multicolumn{4}{|l|}{$\begin{array}{l}\text { Treatment regimen, } \\
n(\%)\end{array}$} \\
\hline Surgery & $17(94.4)$ & $194(95.1)$ & - \\
\hline CRT + surgery & $1(5.6)$ & $10(4.9)$ & - \\
\hline \multicolumn{4}{|l|}{ Recurrence, $n(\%)$} \\
\hline No & $9(50.0)$ & $142(69.3)$ & - \\
\hline Yes & $9(50.0)$ & $63(30.7)$ & - \\
\hline I & $2(22.2)$ & $6(9.5)$ & - \\
\hline II & $1(11.1)$ & $8(12.7)$ & - \\
\hline III & $6(66.7)$ & $33(52.4)$ & - \\
\hline IV & $0(0.0)$ & $16(25.4)$ & - \\
\hline \multicolumn{4}{|l|}{ Metastasis, $n(\%)^{*}$} \\
\hline No & $1(11.1)$ & $4(6.3)$ & - \\
\hline Yes & $8(88.9)$ & $59(93.7)$ & - \\
\hline I & $1(12.5)$ & $3(5.1)$ & - \\
\hline II & $1(12.5)$ & $8(13.6)$ & - \\
\hline III & $6(75.0)$ & $32(54.2)$ & - \\
\hline IV & $0(0.0)$ & $16(27.1)$ & - \\
\hline $\begin{array}{l}\text { MFT in months, } \\
\text { median (range) }\end{array}$ & $\begin{array}{l}64.1 \\
(7.6-128.5)\end{array}$ & $\begin{array}{l}46.7 \\
(0.1-155.0)\end{array}$ & - \\
\hline \multicolumn{4}{|l|}{$\begin{array}{c}\text { Smoking status, } \\
n(\%)\end{array}$} \\
\hline Never & $10(55.6)$ & $100(48.8)$ & - \\
\hline Former & $6(33.3)$ & $70(34.1)$ & - \\
\hline Current & $2(11.1)$ & $35(17.1)$ & - \\
\hline \multicolumn{4}{|l|}{$\begin{array}{l}\text { Hypertension, } \\
n(\%)\end{array}$} \\
\hline Yes & $5(27.8)$ & $99(48.3)$ & - \\
\hline No & $13(72.2)$ & $106(51.7)$ & - \\
\hline \multicolumn{4}{|l|}{$\begin{array}{c}\text { Body mass index, } \\
n(\%)\end{array}$} \\
\hline$<30 \mathrm{~kg} / \mathrm{m}^{2}$ & $8(44.4)$ & $98(47.8)$ & - \\
\hline$\geq 30 \mathrm{~kg} / \mathrm{m}^{2}$ & $7(38.9)$ & $95(46.3)$ & - \\
\hline Unknown & $3(16.7)$ & $12(5.9)$ & - \\
\hline
\end{tabular}

*Number of patients with local recurrence whose disease progressed to metastasis.

-, no data; ccRCC, clear cell renal cell carcinoma; CRT, chemoradiotherapy; MDA, MD Anderson Cancer Center; MFT, median follow-up time; TCGA, The Cancer Genome Atlas database. 
Table 2 miRNAs Differentially Expressed in Tumor and Normal Tissue Pairs in the MDA Discovery, MDA Validation, and TCGA Validation Sets

\begin{tabular}{|c|c|c|c|c|c|c|c|c|}
\hline \multirow[b]{2}{*}{$\operatorname{miR}$} & \multicolumn{4}{|c|}{ MDA discovery } & \multicolumn{4}{|c|}{ MDA validation } \\
\hline & $\begin{array}{l}\text { Tumor, } \\
\text { median }\end{array}$ & $\begin{array}{l}\text { Normal, } \\
\text { median }\end{array}$ & $\begin{array}{l}\text { Fold } \\
\text { change }\end{array}$ & $P$ value* & $\begin{array}{l}\text { Tumor, } \\
\text { median }\end{array}$ & $\begin{array}{l}\text { Normal, } \\
\text { median }\end{array}$ & $\begin{array}{l}\text { Fold } \\
\text { change }\end{array}$ & $P$ value* \\
\hline miR-592 & 57.10 & 9.27 & 6.2 & $3.27 \times 10^{-4}$ & 5.07 & 0.92 & 5.5 & $1.32 \times 10^{-9}$ \\
\hline miR-155-5p & 185.92 & 41.41 & 4.5 & $3.86 \times 10^{-4}$ & 1.83 & 0.55 & 3.3 & $<0.001$ \\
\hline$m i R-21-5 p$ & 5017.11 & 1402.99 & 3.6 & $2.33 \times 10^{-4}$ & 2.78 & 0.38 & 7.3 & $<0.001$ \\
\hline miR-223-3p & 550.92 & 233.53 & 2.4 & $2.49 \times 10^{-2}$ & 1.09 & 0.94 & 1.2 & $3.32 \times 10^{-2}$ \\
\hline miR-331-3p & 60.47 & 27.60 & 2.2 & $1.37 \times 10^{-3}$ & 1.22 & 1.06 & 1.2 & $7.50 \times 10^{-5}$ \\
\hline miR-28-3p & 75.88 & 35.95 & 2.1 & $5.68 \times 10^{-3}$ & 1.07 & 0.92 & 1.2 & $9.18 \times 10^{-3}$ \\
\hline miR-181a-3p & 35.71 & 17.86 & 2.0 & $2.49 \times 10^{-2}$ & 1.56 & 1.08 & 1.4 & $9.30 \times 10^{-5}$ \\
\hline $\operatorname{miR}-106 b-5 p$ & 145.18 & 71.47 & 2.0 & $1.85 \times 10^{-3}$ & 1.25 & 0.87 & 1.4 & $6.49 \times 10^{-9}$ \\
\hline miR-532-5p & 61.22 & 96.09 & 0.6 & $1.01 \times 10^{-3}$ & 0.48 & 1.96 & 0.2 & $<0.001$ \\
\hline $\operatorname{miR}-376 c-3 p$ & 29.20 & 47.15 & 0.6 & $2.22 \times 10^{-2}$ & 0.94 & 2.98 & 0.3 & $6.44 \times 10^{-15}$ \\
\hline miR-27b-3p & 793.82 & 1357.89 & 0.6 & $7.40 \times 10^{-3}$ & 0.84 & 1.43 & 0.6 & $8.88 \times 10^{-16}$ \\
\hline $\operatorname{miR}-376 a-3 p$ & 65.35 & 113.74 & 0.6 & $4.75 \times 10^{-2}$ & 0.72 & 1.85 & 0.4 & $4.34 \times 10^{-10}$ \\
\hline miR-335-5p & 22.01 & 40.65 & 0.5 & $7.38 \times 10^{-4}$ & 1.04 & 3.10 & 0.3 & $1.91 \times 10^{-14}$ \\
\hline miR-218-5p & 71.04 & 137.63 & 0.5 & $1.56 \times 10^{-2}$ & 0.55 & 1.70 & 0.3 & $1.55 \times 10^{-15}$ \\
\hline miR-30a-3p & 129.71 & 307.33 & 0.4 & $3.86 \times 10^{-2}$ & 0.61 & 1.60 & 0.4 & $<0.001$ \\
\hline miR-204-5p & 209.13 & 540.51 & 0.4 & $4.34 \times 10^{-3}$ & 0.54 & 2.91 & 0.2 & $<0.001$ \\
\hline miR-127-3p & 24.29 & 64.75 & 0.4 & $1.08 \times 10^{-2}$ & 0.71 & 2.40 & 0.3 & $2.22 \times 10^{-16}$ \\
\hline miR-429 & 46.14 & 126.31 & 0.4 & $1.56 \times 10^{-2}$ & 0.56 & 2.49 & 0.2 & $<0.001$ \\
\hline miR-199a-5p & 145.51 & 405.77 & 0.4 & $1.76 \times 10^{-2}$ & 0.95 & 2.33 & 0.4 & $3.32 \times 10^{-8}$ \\
\hline
\end{tabular}

miR-s are ordered by fold changes in the discovery group.

${ }^{*} P$ value of $<0.001$ denotes significant value below limit estimable by Stata software version 14.0 ; statistical analysis was performed using the Wilcoxon signed-rank test.

\section{Statistical Analysis}

The Wilcoxon signed-rank test was performed to compare normalized miRNA levels between paired ccRCC tumors and adjacent normal tissues. For both the discovery and TCGA data sets, fold change was calculated as the normalized miRNA level in tumor samples divided by the normalized miRNA level in adjacent normal tissues. In the validation set, fold change in expression level was calculated using the $2^{-\Delta \Delta \mathrm{Ct}}$ method: $2^{-\Delta \Delta \mathrm{Ct}}=2^{-[(\mathrm{CtmiRNA} \text { in tumor }-\mathrm{CtRNU} 44 \text { in }}$ tumor) - (CtmiRNA in normal - CtRNU44 in normal)]

The rank sum test was applied to evaluate differences in median miRNA expression levels in patients with and without recurrence. The associations of miRNA expression levels with recurrence-free survival durations were estimated as hazard ratios and $95 \%$ CIs using Cox proportional hazards regression models and adjusted for potential confounders based on a priori knowledge. Recurrence-free survival was defined as the period from the date of diagnosis to the date of the first event of either recurrence or death. The Kaplan-Meier method was used to estimate recurrence-free survival, and statistical significance was determined using the log-rank test. miRNA expression levels were classified as high or low using the median value in patients without recurrence as the cutpoint. The false-discovery rate was used to account for multiple testing. The combined 2-miRNA risk score in each subject was calculated by a linear combination of the product of reference-normalized expression level of each miRNA by its Cox regression corresponding coefficient. All subjects were assigned to one of two risk groups based on the median risk score; individuals with a risk score higher or lower than the median value were classified as high or low risk, respectively. Statistical analyses were performed using Stata software version 14.0 (Stata Corp., College Station, TX) and R software version 3.01 (The R Foundation for Statistical Computing, Vienna, Austria; https://www.r-project.org, last accessed May 23, 2018). The heat maps were generated using GenePattern software version 3.1 (Broad Institute, Cambridge, MA). All statistical tests were two-sided, and $P$ values of $<0.05$ were considered statistically significant. 
Table 2 (continued)

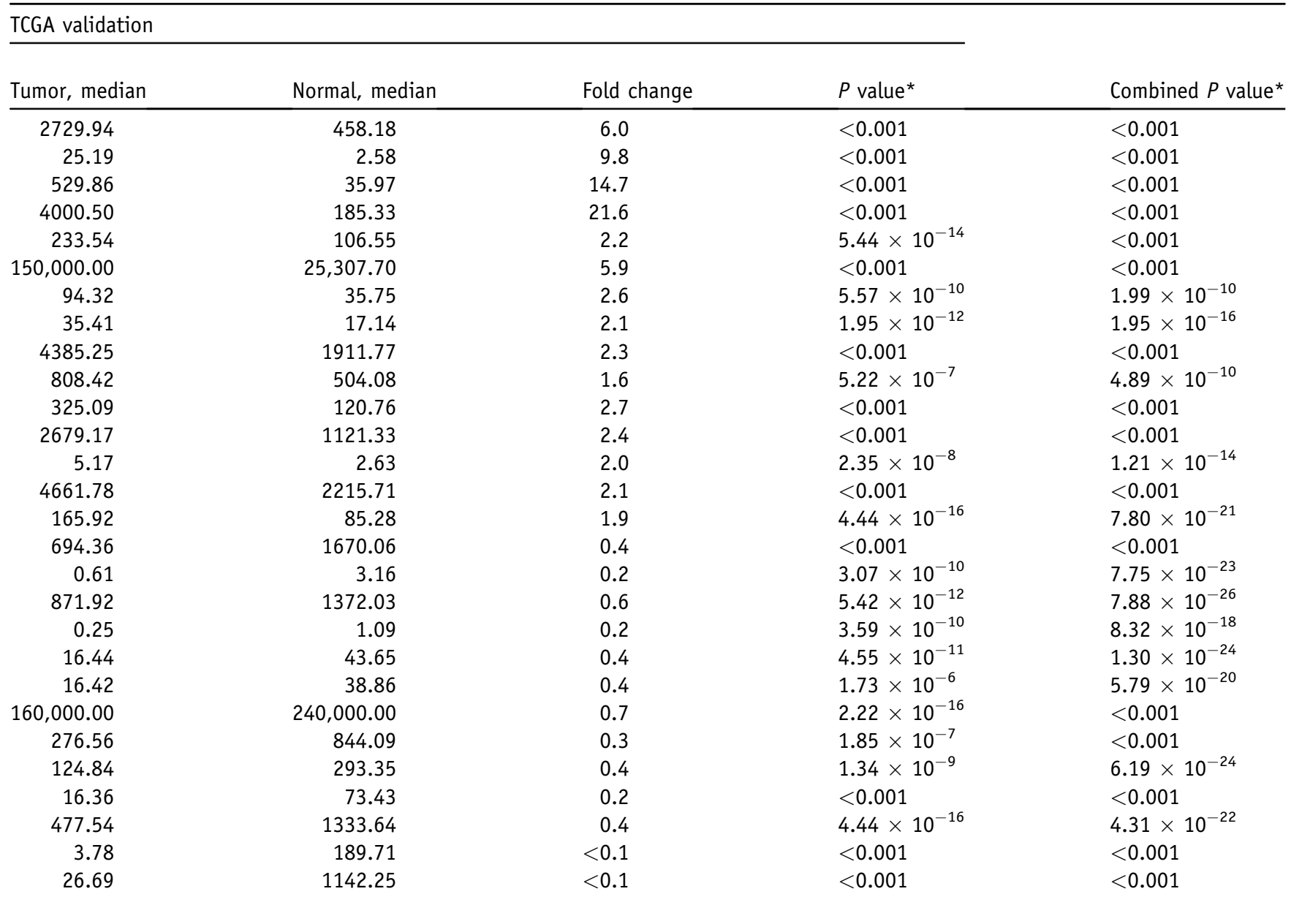

\section{Results}

\section{Patient Characteristics}

This study enrolled 223 ccRCC patients recruited from MDA. The median follow-up time was 46.7 months (range, 0.1 to 155 months). The characteristics of the patients in the discovery and validation cohorts, including age, sex, disease stage, treatment, recurrence, and median follow-up time, are summarized in Table 1 . The majority of the patients $(61.1 \%$ in the discovery set, $60.5 \%$ in the MDA validation set, and $73.8 \%$ in the TCGA validation set) were male, which was consistent with the predominance of men among RCC patients. Of the study population from MDA, 211 patients underwent surgery and 11 were treated with concurrent chemoradiotherapy followed by surgery. In the discovery cohort, 9 patients $(50.0 \%)$ experienced disease recurrence, whereas 63 patients $(30.7 \%)$ in the MDA validation cohort experienced disease recurrence. In both cohorts, most $(>66 \%)$ of the recurrent cases were in patients with late-stage (III and IV) disease, and 9 of 10 patients with local recurrence progressed to metastatic disease [89\% (8/9) and $94 \%(59 / 63)$ in the discovery and validation sets, respectively] (Table 1 ).

\section{miRNAs Differentiating ccRCC Tumor-Normal Pairs}

\section{Discovery Cohort}

Eighteen pairs of ccRCC tumor-tissue and adjacent normal-tissue samples in the discovery cohort were analyzed for the expression of 800 miRNAs using the NanoString platform. Of these, 124 miRNAs were found to be differentially expressed in the 18 ccRCC tumor samples as compared with their adjacent normal tissues $(P<0.05)$ (Supplemental Table S3). Among these, 51 miRNAs showed differential expression, with a false-discovery rate of $<0.1$ (data not shown). The fold changes in miRNA expression levels ranged from 0.119 to 6.312 , with miR$200 c-3 p$ being the most down-regulated miRNA and miR-142-3p being the most up-regulated (Supplemental Table S3). 


\section{Validation Cohort}

The top 56 most differentially expressed miRNAs in the discovery cohort were then validated in an independent data set of 205 ccRCC tumor-normal tissue pairs using the Fluidigm 96.96 Dynamic Array and Biomark platform. Of these, 31 miRNAs were confirmed to be significantly dysregulated in the ccRCC tumor samples compared with their adjacent normal tissues with patterns of up- and downregulation similar to those in the discovery data set $(P<0.05)$ (Supplemental Table S4). The fold changes ranged from 0.008 to 7.316 , with $\mathrm{miR}-141-3 \mathrm{p}$ being the most down-regulated miRNA and miR-210-3p being the most up-regulated.

\section{External Validation Using the TCGA Data Set}

The 31 miRNAs identified in the MDA validation set were further tested using an independent data set of $68 \mathrm{ccRCC}$ normal-tumor pairs from the TCGA database. Twenty-eight of the 31 miRNAs in TCGA tumors were significantly dysregulated, exhibiting up- or down-regulation patterns similar to those in the MDA cohorts. The fold changes ranged from $<0.1$ to 21.6 , with miR-141 being the most down-regulated miRNA and miR-210-3p being the most upregulated. The final miRNA signature consisted of 15 miRNAs that were up-regulated and 13 miRNAs that were down-regulated across the three independent data sets. These miRNAs and their combined (meta-analysis) $P$ values are shown in Table 2. Unsupervised heat maps of these differentially expressed miRNAs are displayed in Supplemental Figures S2-S4.

\section{miRNAs Associated with ccRCC Recurrence}

\section{Discovery}

To identify potential miRNA markers of tumor recurrence, a discovery cohort of 18 ccRCC tumor-tissue samples (9 recurrent and 9 nonrecurrent) were analyzed for the expression of 800 human miRNAs using the NanoString platform. Because the sample size of this cohort was small, miRNAs were considered to be differentially expressed in tumor and adjacent normal tissue if $P<0.1$ for further validation. Using this cutoff and the rank sum test, 23 miRNAs were found to be significantly differentially expressed in the recurrence and nonrecurrence groups (Supplemental Table S5), with 21 up-regulated miRNAs and 2 down-regulated miRNAs. The fold changes ranged from 0.085 to 3.582 , with miR-204-5p being the most down-regulated miRNA and miR-155-5p being the most upregulated miRNA in recurrent tumors.
A

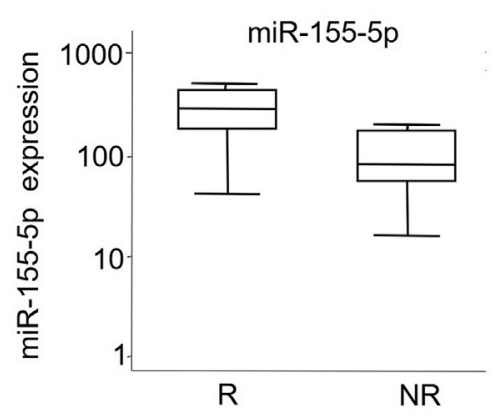

D

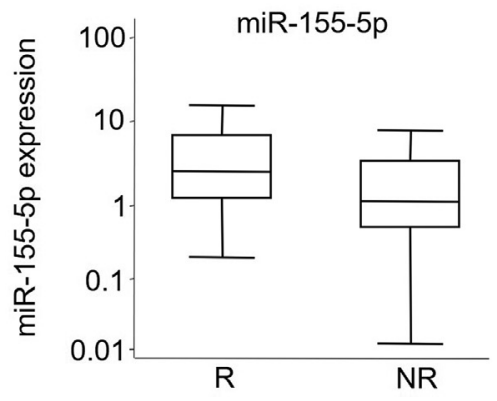

B

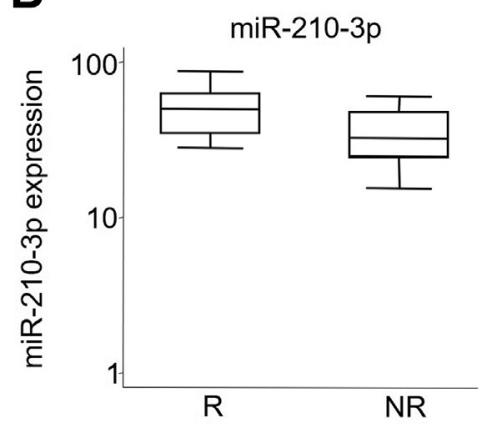

E

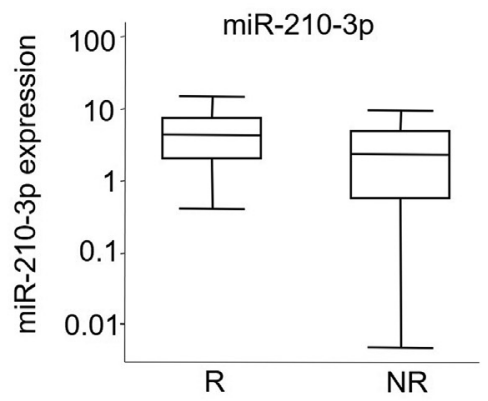

C

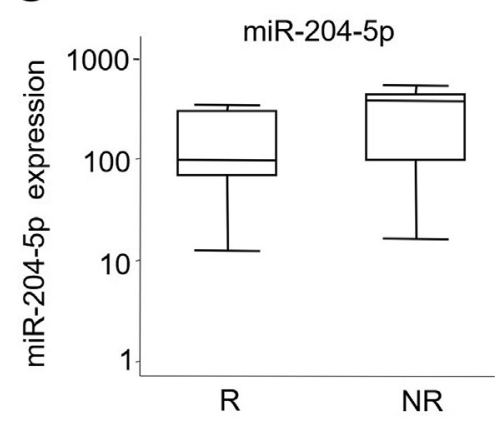

$\mathbf{F}$

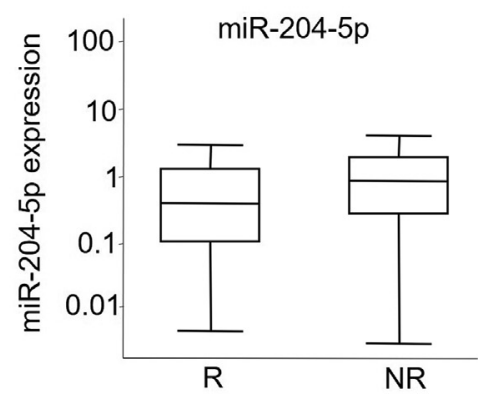

Figure 1 Box plots showing dysregulation of miR-155-5p, miR-210-3p, and miR-204-5p in the tumor tissues of patients with recurrent clear-cell renal cell carcinoma. Expression levels (shown on a $\log _{10}$ scale on the $y$ axes) of candidate miRNA biomarkers in the discovery $(\mathbf{A}-\mathbf{C})$ and validation (D-F) sets. Levels of miR-155a-5p, miR-204-5p, and miR-210-3p in tumor tissue were compared between patients with recurrent (R) and nonrecurrent (NR) disease. The lines inside the boxed areas denote the medians; boxed areas define the interquartile range of values; whiskers define the $95 \%$ CIs. $P=0.038(\mathbf{A}) ; P=0.058$ (B); $P=0.085(\mathbf{C}) ; P=0.001$ (D); $P=0.003(\mathbf{E}) ;$ and $P=0.014$ (F) (rank sum test for all). 
Table 3 miRNAs Associated with ccRCC Recurrence in the MDA Validation Set

\begin{tabular}{llll}
\hline & Up- or down-regulation & & \\
miRNA & in recurrent tumors & $\mathrm{HR}^{* \dagger}(95 \% \mathrm{CI})$ & $P$ value \\
\hline miR-155-5p & Up & $2.64(1.49-4.70)$ & 0.0009 \\
miR-210-3p & Up & $1.80(1.04-3.12)$ & 0.036 \\
miR-204-5p & Down & $0.59(0.35-1.02)$ & 0.059 \\
\hline
\end{tabular}

*The reference group was low expression level. Levels were dichotomized using the median expression level in nonrecurrent tumor tissues as the cut point.

${ }^{\dagger}$ Calculated by univariable Cox regression model.

ccRCC, clear cell renal cell carcinoma; HR, hazard ratio; MDA, MD Anderson Cancer Center.

\section{Validation}

Eight miRNAs (miR-155-5p, miR-18a-5p, miR-192-5p, miR-204-5p, miR-210-3p, miR-27b-3p, miR-28-5p, and miR-34a-5p) from the 23 miRNAs that showed differential expression between the recurrence and nonrecurrence groups were further selected to be measured in the validation cohort of 205 patients. These 8 miRNAs also displayed consistent differential expression between tumor and adjacent normal tissues (Supplemental Tables S3, S4 and S5). Results showed that three miRNAs were validated. miR155-5p and miR-210-3p were confirmed to be significantly up-regulated, and miR-204-5p was significantly down-regulated, in recurrent tumors compared to nonrecurrent tumors in both the discovery set (Figure 1, A-C) and the validation set (Figure 1, D-F). Furthermore, a high level of miR-155-5p was associated with a significant, 2.64fold, increased risk for ccRCC recurrence $(95 \% \mathrm{CI}$, 1.49-4.70; $P=0.0009)$ in the MDA validation set analyzed with the univariate model (Table 3). Data from the discovery set were not analyzed due to the underpowered sample size. A similar result was found with miR-210-3p (hazard ratio, 1.80; 95\% CI, 1.04-3.12; $P=0.036$ ), whereas a high level of miR-204-5p was borderline protective with a nonsignificant association (hazard ratio, 0.59; 95\% CI, 0.35-1.02; $P=0.059$ ). Kaplan-Meier analysis showed that patients in the validation set with a high miR$155-5 p$ level had significantly shorter median recurrencefree survival times than did those with a low level $[P=0.0001$ (log rank) (Figure 2). A similar decrease in recurrence-free survival time was also observed in patients with a high level of miR-210-3p. Thus, a risk score based on miR-155-5p and miR-210-3p expression levels was designed and was used to stratify our validation cohort into high-, intermediate-, and low-risk groups $[P=0.0004$ (log rank)] (Supplemental Figure S5). Both univariate and multivariate Cox regression analyses in the validation cohort demonstrated that the risk for recurrence increased as the risk score increased $(P$ for trend, $<0.01)$ (Table 4$)$.

miRNA Pathway Analysis

To explore the biological mechanisms that involved these miRNAs in RCC tumorigenesis, target prediction analysis was performed, coupled with pathway analysis. Target genes of the 28 differentially expressed miRNAs in ccRCC tumors were identified using the miRsystem database. Pathway analysis showed that most of these miRNAs were enriched in P53-independent and P53-dependent DNAdamage response, regulation of cell cycle G1/S, apoptosis, and wingless-type mouse mammary tumor virus integration site family (WNT) signaling pathways (Supplemental Table S6).

Analysis of the pathways involving miR-155-5p and miR-210-3p, high levels of which were associated with ccRCC recurrence, showed that inflammation-related pathways, such as IL-12-, IL-4-, IL-3-, and IL-6-mediated signaling, and IL-2 signaling events mediated by phosphoinositide 3-kinase, were enriched. In addition, miR$155-5 p$ and miR-210-3p were involved in several other critical pathways, such as B cell-receptor signaling and class 1 phosphoinositide 3-kinase signaling (Supplemental Table S7).

\section{Discussion}

In this multiphase study, 28 significantly dysregulated miRNAs (15 up-regulated and 13 down-regulated) were compared between ccRCC tumor samples and paired adjacent normal-tissue samples. Furthermore, 2 miRNAs, miR-155-5p and miR-210-3p, were identified as being associated with tumor recurrence in ccRCC patients; risk scores, with the potential for use in classifying patients based on risk their for ccRCC recurrence, was devised for these miRNAs.

This study replicated the results from previous studies that also included both discovery and validation sets. For instance, several investigations found up-regulation of miR21-5p, miR-142, miR-155-5p, miR-210-3p, miR-106b-5p, and miR-34a-5p and down-regulation of miR-532-5p, miR-204-5p, miR-27b-3p, miR-141-3p, miR-200c-3p, miR-127-3p, miR-429, and miR-199a-5p in ccRCC. $^{9-13,24-26}$ Moreover, significant dysregulation of several other miRNAs, including up-regulation of miR-935p, miR-592, miR-331-3p, miR-28-3p, miR-25-3p, miR223-3p, miR-181a-5p, and miR-15b-5p and downregulation of miR-335-5p, miR-218-5p, miR-30a-3p, and miR-376a-3p, had not been previously reported. Dysregulation of these miRNAs in both discovery and validation cohorts was confirmed.

To elucidate the biological functions of these dysregulated miRNAs, their putative target genes were identified and their related pathways were analyzed using the miRsystem database. Pathway analysis revealed that most of these dysregulated miRNAs were enriched in known pathways involved in cancer - P53-dependent and P53-independent DNA-damage response, regulation of cell-cycle G1/S, apoptosis, and WNT/ $\beta$-catenin signaling - suggesting crucial roles of these miRNAs and pathways in the pathogenesis of ccRCC. The precise 

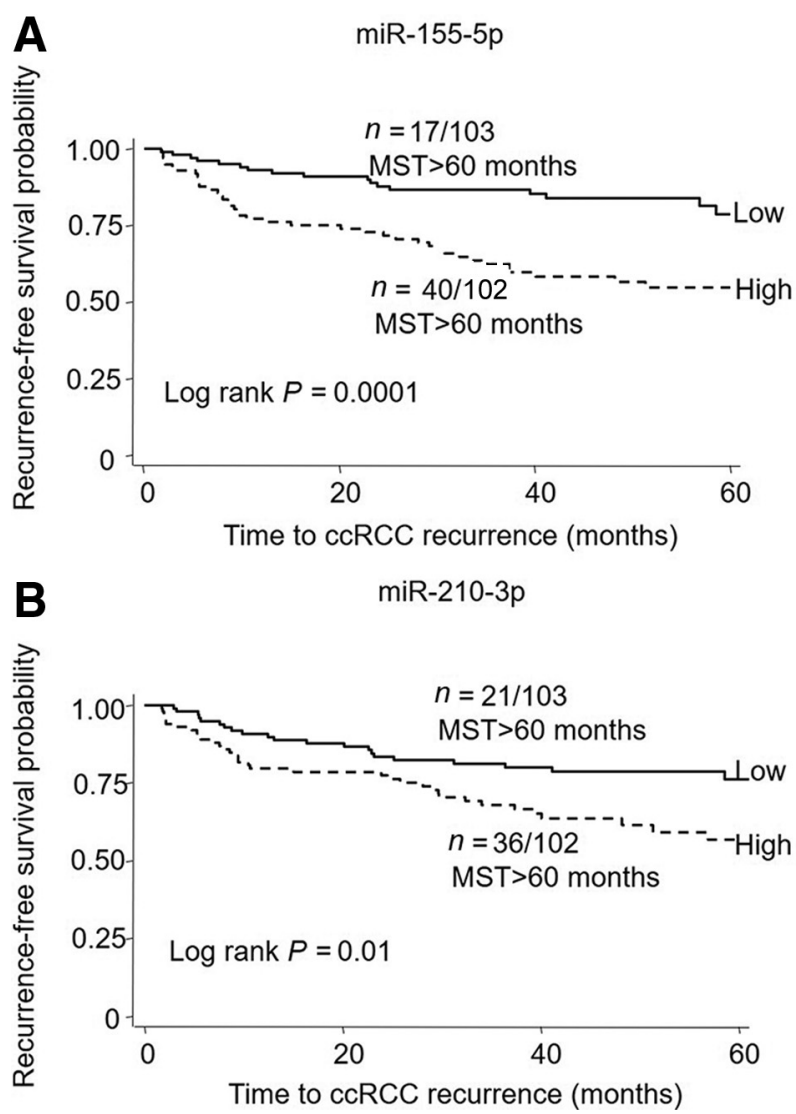

Figure 2 Kaplan-Meier curves comparing median recurrence-free survival times (MST) in patients with clear-cell renal cell carcinoma (ccRCC) and low versus high levels of miR-155-5p (A) and miR-210-3p (B) in the MD Anderson Cancer Center (MDA) validation set. $\mathrm{N}$, [Number of patients with an event (recurrence)]/[Total number of patients in the data set].

mechanisms of these miRNAs in RCC development remain to be elucidated by functional experiments.

Two miRNAs that predicted ccRCC recurrence, miR155-5p and miR-210-3p, were identified. Up-regulation of miR-155-5p was commonly found in multiple types of cancer, including RCC. ${ }^{9,12,13}$ In this study, it was confirmed that miR-155-5p levels were much higher in renal tumor tissues compared with normal tissues. Furthermore, it was found for the first time that up-regulation of miR-155-5p was correlated with ccRCC recurrence. Reports have illustrated the important roles of miR-155-5p in several biological functions, including cell proliferation, apoptosis, migration, invasion, hematopoiesis, and immune regulation. ${ }^{27-29}$ miR-155-5p also targets $B A C H 1^{30}$ and $E 2 F 2^{31}$ in RCC, genes involved in transcriptional regulation and cell cycle, respectively. Another potential target for miR$155-5 \mathrm{p}$ is the hypoxia-inducible factor- $1 \alpha$ gene $(H I F 1 A),{ }^{32}$ which plays an essential role in tumor angiogenesis and progression. Our study findings support the potential oncogenic role of miR-155-5p in RCC tumorigenesis and identified the novel role of this miRNA in RCC recurrence.

miR-210-3p is overexpressed in many kinds of human malignancies, including RCC, suggesting that it plays an important role in tumorigenesis. In this study, it was confirmed that miR-210-3p was expressed at a higher level in tumor tissues than in adjacent normal tissues from RCC patients, which is consistent with previous findings. ${ }^{9,11,33,34}$ In addition, higher miR-210-3p levels were found in the tumor tissues of patients who had recurrent disease than in the tissues of patients whose disease did not recur. Similarly, Neal et $\mathrm{al}^{35}$ determined that higher miR-210-3p levels in renal tumor tissues were associated with shorter patient survival times. Although the mechanism by which miR-210-3p contributes to ccRCC is not yet clear, one event known to be involved in ccRCC development is frequent mutations in the $V H L$ gene, which causes the accumulation of hypoxia inducible factor. ${ }^{36}$ Previous research has shown that overexpression of miR-210-3p was related to an active hypoxia inducible factor-signaling pathway. For instance, Camps et $\mathrm{al}^{37}$ demonstrated that overexpression of miR210-3p in breast tumor tissue was induced by hypoxia in a hypoxia inducible factor- $1 \alpha-$ and Von Hippel-Lindau tumor suppressor-dependent fashion. In RCC, increased miR-210 levels were correlated with the expression of a hypoxia inducible factor-regulated gene, carbonic anhydrase IX, and also associated with VHL mutation or promoter methylation. ${ }^{35}$ Further studies are necessary to reveal the exact mechanism by which miR-210-3p affects ccRCC tumorigenesis and progression.

Our pathway analysis showed that miR-155-5p and miR210-3p were enriched in inflammatory cytokine-related pathways, such as IL-4- and IL-12-mediated signaling. miR-155-5p was also involved in IL-6-, IL-2-, and IL-3-mediated signaling. It is widely believed that chronic inflammation is an important driver of tumor development, progression, and metastasis. ${ }^{38}$ The proteins produced during chronic inflammation, including cytokines, chemokines, matrix-degrading enzymes, and growth factors, may damage DNA and affect the capacity of cells to survive and proliferate, thereby promoting tumor initiation, growth, and metastasis. ${ }^{39,40}$ Infiltrating lymphocytes and inflammatory

Table 4 Risk Group Stratification Using Risk Score Generated from miR-155-5p and miR-210-3p in the MDA Validation Set

\begin{tabular}{lccc}
\hline Risk score & $\mathrm{HR}^{\dagger}(95 \% \mathrm{CI})$ & $P$ value & $P$ trend \\
\hline $\begin{array}{l}\text { Univariate } \\
\text { Low }\end{array}$ & Reference & & \\
$\quad$ Intermediate & $1.9(0.87-4.12)$ & 0.107 & \\
$\quad$ High & $3.36(1.61-6.99)$ & 0.001 & 0.0007 \\
$\begin{array}{l}\text { Multivariate } \\
\quad \text { Low }\end{array}$ & & \\
Intermediate & $1.39(0.64-3.06)$ & 0.406 & \\
High & $2.7(1.28-5.68)$ & 0.009 & 0.005 \\
\hline
\end{tabular}

*Low, low levels of both miR-155-5p and miR-210-3p; intermediate, high level of either miR-155-5p or miR-210-3p; high, high levels of both miRNAs.

${ }^{\dagger}$ Multivariate model adjusted for age, sex, clinical stage, smoking, hypertension, and obesity (body mass index $<30$ vs $\geq 30$ ).

HR, hazard ratio; MDA, MD Anderson Cancer Center. 
cytokines make up the complex tumor-immune microenvironment. The interaction between tumor cells and the immune cells in the tumor microenvironment may play a crucial role in the growth and recurrence of RCC. Therefore, modulating these immunosuppressive factors in the tumor microenvironment may be a therapeutic strategy in RCC. Our results suggest that miR-155-5p and miR-210-3p might be involved in regulating the tumor microenvironment during RCC recurrence. However, the functional mechanisms of these miRNAs in recurrent and metastatic RCC require further characterization by biological experiments.

To our knowledge, only a small number of miRNAs have been previously reported to be related to ccRCC recurrence, including miR-106b, miR-126, miR-139-5p, miR-143, miR26a, miR-145, miR-10b, miR-195, miR-126, miR-127, miR-204-5p, and miR-210. ${ }^{9,11,16-18}$ However, of these miRNAs, only miR-204-5p and miR-210 were replicated in this study, the former with borderline significance. Differences in methodology, such as assay platforms used, experimental conditions, subtypes of renal cancer analyzed, sample size, and other statistical considerations, might contribute to the variations in the results. ${ }^{12}$ Our previous study has reported the association between a decreased level of miR-204-5p and shorter RCC recurrence-free survival, and the link between miR-204-5p and ccRCC recurrence might be mediated by the regulation of the expression of obesity-related target genes.

In this study, miRNA expression was examined in RCC tumor and normal tissues. Since tumors may secrete miRNAs in exosomes, circulating miRNAs have been explored as potential noninvasive diagnostic and prognostic markers of different cancer sites, including RCC. For example, several studies have found serum miR-210 as a potential diagnostic marker of ccRCC, ${ }^{41-43}$ which is consistent with our finding of elevated miR-210 expression in tumor tissues and the potential role of this miRNA as a predictor of RCC recurrence. Future independent prospective studies are necessary to assess the utility of circulating miRNAs for clinical application.

Due to the usage of different gene expression platforms, the data from the discovery and validation sets could not be merged. Nonetheless, it has been shown previously ${ }^{44}$ and verified by us during the present study that there was good correlation (Spearman correlation $r \approx 0.4, P<0.01$ ) between the NanoString and Fluidigm real-time quantitative PCR data, suggesting the validity of the approach. It should be mentioned that the small sample size of the discovery set might have limited the power of the present study in identifying more differentially expressed miRNAs during the screening stage. Therefore caution should be used regarding the interpretation of negative data.

In conclusion, this study identified 28 miRNAs that were dysregulated in the tumor tissues of ccRCC patients. Furthermore, miR-155-5p and miR-210-3p were shown to be potential predictors of tumor recurrence in patients with RCC. The combined sample size of this study was relatively large as compared with those of previous studies. ${ }^{9-13,16-18}$ Moreover, an independent data set was used to validate our findings, and both global and targeted approaches involving two methodologies were utilized to profile tissue miRNAs. The candidate miRNAs were significant in univariate and multivariate models, suggesting that their associations were less likely to have been confounded by host and clinical factors. Overall, the findings from this study suggest that miR-155-5p and miR-210-3p may possess translational potential as clinical biomarkers for use in identifying ccRCC patients at high risk for recurrence. These findings also provide potential insight into the biological mechanisms that link inflammation and ccRCC recurrence.

\section{Supplemental Data}

Supplemental material for this article can be found at https://doi.org/10.1016/j.ajpath.2018.07.026.

\section{References}

1. Gupta K, Miller JD, Li JZ, Russell MW, Charbonneau C: Epidemiologic and socioeconomic burden of metastatic renal cell carcinoma (mRCC): a literature review. Cancer Treat Rev 2008, 34:193-205

2. Rini BI, Campbell SC, Escudier B: Renal cell carcinoma. Lancet 2009, 373:1119-1132

3. Ha SC, Zlomke HA, Cost N, Wilson S: The past, present, and future in management of small renal masses. J Oncol 2015, 2015:364807

4. Nerich V, Hugues M, Paillard MJ, Borowski L, Nai T, Stein U, Nguyen Tan Hon T, Montcuquet P, Maurina T, Mouillet G, Kleinclauss F, Pivot X, Limat S, Thiery-Vuillemin A: Clinical impact of targeted therapies in patients with metastatic clear-cell renal cell carcinoma. Onco Targets Ther 2014, 7:365-374

5. Pichler M, Hutterer GC, Chromecki TF, Jesche J, Kampel-Kettner K, Rehak P, Pummer K, Zigeuner R: External validation of the Leibovich prognosis score for nonmetastatic clear cell renal cell carcinoma at a single European center applying routine pathology. J Urol 2011, 186: 1773-1777

6. Hsieh JJ, Purdue MP, Signoretti S, Swanton C, Albiges L, Schmidinger M, Heng DY, Larkin J, Ficarra V: Renal cell carcinoma. Nat Rev Dis Primers 2017, 3:17009

7. Bartel DP: MicroRNAs: genomics, biogenesis, mechanism, and function. Cell 2004, 116:281-297

8. Lin S, Gregory RI: MicroRNA biogenesis pathways in cancer. Nat Rev Cancer 2015, 15:321-333

9. Shu X, Hildebrandt MA, Gu J, Tannir NM, Matin SF, Karam JA, Wood CG, Wu X: MicroRNA profiling in clear cell renal cell carcinoma tissues potentially links tumorigenesis and recurrence with obesity. Br J Cancer 2017, 116:77-84

10. He H, Wang L, Zhou W, Zhang Z, Wang L, Xu S, Wang D, Dong J, Tang C, Tang H, Yi X, Ge J: MicroRNA expression profiling in clear cell renal cell carcinoma: identification and functional validation of key miRNAs. PLoS One 2015, 10:e0125672

11. Osanto S, Qin Y, Buermans HP, Berkers J, Lerut E, Goeman JJ, van Poppel H: Genome-wide microRNA expression analysis of clear cell renal cell carcinoma by next generation deep sequencing. PLoS One 2012, 7:e38298

12. White NM, Bao TT, Grigull J, Youssef YM, Girgis A, Diamandis M, Fatoohi E, Metias M, Honey RJ, Stewart R, Pace KT, Bjarnason GA, Yousef GM: miRNA profiling for clear cell renal cell carcinoma: biomarker discovery and identification of potential controls and consequences of miRNA dysregulation. J Urol 2011, 186:1077-1083 
13. Jung M, Mollenkopf HJ, Grimm C, Wagner I, Albrecht M, Waller T, Pilarsky C, Johannsen M, Stephan C, Lehrach H, Nietfeld W, Rudel T, Jung K, Kristiansen G: MicroRNA profiling of clear cell renal cell cancer identifies a robust signature to define renal malignancy. J Cell Mol Med 2009, 13:3918-3928

14. Cancer Genome Atlas Research Network: Comprehensive molecular characterization of clear cell renal cell carcinoma. Nature 2013, 499:43-49

15. Slaby O, Jancovicova J, Lakomy R, Svoboda M, Poprach A, Fabian P, Kren L, Michalek J, Vyzula R: Expression of miRNA-106b in conventional renal cell carcinoma is a potential marker for prediction of early metastasis after nephrectomy. J Exp Clin Cancer Res 2010, 29:90

16. Slaby O, Redova M, Poprach A, Nekvindova J, Iliev R, Radova L, Lakomy R, Svoboda M, Vyzula R: Identification of MicroRNAs associated with early relapse after nephrectomy in renal cell carcinoma patients. Genes Chromosomes Cancer 2012, 51:707-716

17. Nakata W, Uemura M, Sato M, Fujita K, Jingushi K, Ueda Y, Kitae K, Tsujikawa K, Nonomura N: Expression of miR-27a-3p is an independent predictive factor for recurrence in clear cell renal cell carcinoma. Oncotarget 2015, 6:21645-21654

18. Samaan S, Khella HW, Girgis A, Scorilas A, Lianidou E, Gabril M, Krylov SN, Jewett M, Bjarnason GA, El-said H, Yousef GM: miR-210 is a prognostic marker in clear cell renal cell carcinoma. J Mol Diagn 2015, 17:136-144

19. Kozomara A, Griffiths-Jones S: miRBase: annotating high confidence microRNAs using deep sequencing data. Nucleic Acids Res 2014, 42: D68-D73

20. Clague J, Lin J, Cassidy A, Matin S, Tannir NM, Tamboli P, Wood CG, Wu X: Family history and risk of renal cell carcinoma: results from a case-control study and systematic meta-analysis. Cancer Epidemiol Biomarkers Prev 2009, 18:801-807

21. Livak KJ, Schmittgen TD: Analysis of relative gene expression data using real-time quantitative PCR and the 2(-Delta Delta C(T)) Method. Methods 2001, 25:402-408

22. Kroh EM, Parkin RK, Mitchell PS, Tewari M: Analysis of circulating microRNA biomarkers in plasma and serum using quantitative reverse transcription-PCR (qRT-PCR). Methods 2010, 50:298-301

23. Lu TP, Lee CY, Tsai MH, Chiu YC, Hsiao CK, Lai LC, Chuang EY: miRSystem: an integrated system for characterizing enriched functions and pathways of microRNA targets. PLoS One 2012, 7:e42390

24. Juan D, Alexe G, Antes T, Liu H, Madabhushi A, Delisi C, Ganesan S, Bhanot G, Liou LS: Identification of a microRNA panel for clear-cell kidney cancer. Urology 2010, 75:835-841

25. Nakada C, Matsuura K, Tsukamoto Y, Tanigawa M, Yoshimoto T, Narimatsu T, Nguyen LT, Hijiya N, Uchida T, Sato F, Mimata H, Seto M, Moriyama M: Genome-wide microRNA expression profiling in renal cell carcinoma: significant down-regulation of miR-141 and miR-200c. J Pathol 2008, 216:418-427

26. Yi Z, Fu Y, Zhao S, Zhang X, Ma C: Differential expression of miRNA patterns in renal cell carcinoma and nontumorous tissues. J Cancer Res Clin Oncol 2010, 136:855-862

27. He XH, Zhu W, Yuan P, Jiang S, Li D, Zhang HW, Liu MF: miR-155 downregulates ErbB2 and suppresses ErbB2-induced malignant transformation of breast epithelial cells. Oncogene 2016, 35:6015-6025

28. Zonari E, Pucci F, Saini M, Mazzieri R, Politi LS, Gentner B, Naldini L: A role for miR-155 in enabling tumor-infiltrating innate immune cells to mount effective antitumor responses in mice. Blood $2013,122: 243-252$
29. Ji J, Zheng X, Forgues M, Yamashita T, Wauthier EL, Reid LM, Wen X, Song Y, Wei JS, Khan J, Thorgeirsson SS, Wang XW: Identification of microRNAs specific for epithelial cell adhesion molecule-positive tumor cells in hepatocellular carcinoma. Hepatology 2015, 62:829-840

30. Li S, Chen T, Zhong Z, Wang Y, Li Y, Zhao X: microRNA-155 silencing inhibits proliferation and migration and induces apoptosis by upregulating BACH1 in renal cancer cells. Mol Med Rep 2012, 5: 949-954

31. Gao Y, Ma X, Yao Y, Li H, Fan Y, Zhang Y, Zhao C, Wang L, Ma M, Lei Z, Zhang X: miR-155 regulates the proliferation and invasion of clear cell renal cell carcinoma cells by targeting E2F2. Oncotarget 2016, 7:20324-20337

32. Bruning U, Cerone L, Neufeld Z, Fitzpatrick SF, Cheong A, Scholz CC, Simpson DA, Leonard MO, Tambuwala MM, Cummins EP, Taylor CT: MicroRNA-155 promotes resolution of hypoxia-inducible factor 1alpha activity during prolonged hypoxia Mol Cell Biol 2011, 31:4087-4096

33. Gu L, Li H, Chen L, Ma X, Gao Y, Li X, Zhang Y, Fan Y, Zhang X: MicroRNAs as prognostic molecular signatures in renal cell carcinoma: a systematic review and meta-analysis. Oncotarget 2015, 6: 32545-32560

34. Redova M, Poprach A, Besse A, Iliev R, Nekvindova J, Lakomy R, Radova L, Svoboda M, Dolezel J, Vyzula R, Slaby O: MiR-210 expression in tumor tissue and in vitro effects of its silencing in renal cell carcinoma. Tumour Biol 2013, 34:481-491

35. Neal CS, Michael MZ, Rawlings LH, Van der Hoek MB, Gleadle JM: The VHL-dependent regulation of microRNAs in renal cancer. BMC Med 2010, 8:64

36. Cohen HT, McGovern FJ: Renal-cell carcinoma. N Engl J Med 2005 , $353: 2477-2490$

37. Camps C, Buffa FM, Colella S, Moore J, Sotiriou C, Sheldon H, Harris AL, Gleadle JM, Ragoussis J: hsa-miR-210 Is induced by hypoxia and is an independent prognostic factor in breast cancer. Clin Cancer Res 2008, 14:1340-1348

38. Hanahan D, Weinberg RA: Hallmarks of cancer: the next generation. Cell 2011, 144:646-674

39. Coussens LM, Werb Z: Inflammation and cancer. Nature 2002, 420 : 860-867

40. Grivennikov SI, Greten FR, Karin M: Immunity, inflammation, and cancer. Cell 2010, 140:883-899

41. Zhao A, Li G, Péoc'h M, Genin C, Gigante M: Serum miR-210 as a novel biomarker for molecular diagnosis of clear cell renal cell carcinoma. Exp Mol Pathol 2013, 94:115-120

42. Iwamoto H, Kanda Y, Sejima T, Osaki M, Okada F, Takenaka A: Serum miR-210 as a potential biomarker of early clear cell renal cell carcinoma. Int J Oncol 2014, 44:53-58

43. Fedorko M, Stanik M, Iliev R, Redova-Lojova M, Machackova T, Svoboda M, Pacik D, Dolezel J, Slaby O: Combination of miR-378 and miR-210 serum levels enables sensitive detection of renal cell carcinoma. Int J Mol Sci 2015, 16:23382-23389

44. Kolbert CP, Feddersen RM, Rakhshan F, Grill DE, Simon G, Middha S, Jang JS, Simon V, Schultz DA, Zschunke M, Lingle W, Carr JM, Thompson EA, Oberg AL, Eckloff BW, Wieben ED, Li P, Yang P, Jen J: Multi-platform analysis of microRNA expression measurements in RNA from fresh frozen and FFPE tissues. PLoS One 2013, 8:e52517 